\title{
PHOTOREFRACTIVE KERATECTOMY WITH COLLAGEN CROSS LINKING IN SUSPICIOUS AND EARLY KERATOCONUS
}

\author{
Abdelallem, A. ${ }^{1(*)}$, Abd El-badie, M. $^{1}$ \& Radwan, G. ${ }^{2}$ \\ 1 Ophthalmology dept., Faculty of Medicine, Al-Azhar Univ., Assiut, Egypt \\ 2Ophthalmology dept., Faculty of Medicine, Sohag Univ., Sohag, Egypt \\ *E-mail: Ahmed4eye@yahoo.com
}

\begin{abstract}
Purpose: To study the efficacy and safety of PRK and corneal cross linking (CXL) For treatment of suspicious and early Keratoconus. Methods: fifty eyes of thirty two patients with suspicious and early keratoconus were included. all patient underwent Simultaneous PRK and corneal cross linking (CXL) The outcomes were evaluated at 18 months in all eyes. Result: $80 \%$ percent of UNCVA postoperatively are equal to preoperatively BCVA or gained 1 or more lines $20 \%$ only Lost 1 line, $62 \%$ of the eyes are within 1.0D. of emmetropia. None of the eyes had > $2.0 \mathrm{D}$ and $54 \%$ of the eyes had Astigmatism by K-Reading within 1.0D. None of the eyes had > 2.0D. Conclusion: We concluded that The Combined non-topography-guided PRK and CXL is an effective procedures for correcting mild refractive error and improving visual acuity and safety option for arresting the ectatic progression, with improvement of corneal keratometric values in patients with suspicious and early keratoconus provided that we adhere to the selection criteria of that we recommend. However, future studies with larger cohorts of patients and longer follow-up periods are needed to determine the safety, efficacy, and stability of such procedure.
\end{abstract}

Keywords: Photorefractive Keratectomy, Collagen Cross Linking, keratoconus

\section{Introduction}

Keratoconus is a progressive, noninflammatory, bilateral asymmetricalectatic corneal disease, characterized by paraxial stromal thinning and weakening that leads to corneal surface distortion. Visual loss occurs primarily from irregular astigmatism and myopia, and secondarily from corneal scarring. Protrusion usually but not exclusively affects the axial and inferonasal cornea [1]. Forme Fruste Keratoconus (FFKC) diagnosed when screening patients for LASIK, positive identification of a sub clinical form of keratoconus known as formefruste keratoconus (FFKC) is important. Per forming LASIK on patients with FFKC significantly increases the risk of developing ectasia. Therefore, it is prudent to identify such patients and if appropriate consider alternative corrective procedures such as phakic IOLs (e.g. Staar ICL) or photorefractive keratectomy (PRK) [2]. FFKC differs from keratoconus in that 
the latter can be diagnosed clinically with use of slit lamp examination and characteristic pattern on corneal topography as discussed above. FFKC has no identifiable slit lamp signs, but corneal topography shows a cone pattern. In other words, FFKC is diagnosed by topography alone. FFKC may or may not exhibit astigmatism on manifest refraction and BSCVA may be $20 / 20$ or it may be reduced [3]. Another finding of interest when evaluating a patient for LASIK is the subtle entity known simply as "inferior steepening". Like FFKC, this entity also is strictly a topography diagnosis. Slit lamp exami-nation is unre-markable. Manifest refraction will usually not have any astigmatism and BSCVA is typically 20/20 (assuming no unrelated pathology is present such as amblyopia or macular scarring, for examples). Performing LASIK on these corneas can evoke a higher keratoectasia risk as well. There can be overlap between FFKC and inferior steepening, so these conditions are not mutually exclusive. FFKC topography maps tend to show marked steeper colors such as red, while inferior steepening may have normal curvatures, but the inferior patternis the only unusual sign. 3 Summary

\section{Patients and methods \\ 2.1. Patients}

This study included fifty eyes of 32 patient $(31.2 \%)$ were male and $(68.8 \%)$ were female, fig. (1) the mean age of study pati-ents was $31.2 \pm 4.2$ years old operated on between November 2014 to November 2016 that had simultaneous PRK and CXL in cases of suspicious keratoconic $22 \mathrm{pt}(62.5 \%)$ and early and keratoconic $10 \mathrm{pt}$ (37.5\%). According to $\mathrm{Lu}$ and Azar scoring system 15 cases of suspicious with score of 2 and 7 cases with score of 3 Minimum corneal thickness $485 \mu \mathrm{m}$. Maximum corneal thickness $551 \mu \mathrm{m}$, Keratometic reading between 42.73 and 46.33 Ds. While all 10 cases with earlykeratoconic cases with score of 4, Minimum corneal of top-ographic Criteria of Keratoconus When more than one of the following criteria are found, any of the above mentioned patterns is considered as frank KC, Forme Fruste Keratoconus, (FFKC), early stage Keratoconus, or at least a case of suspicion (according to the severity and amount of signs) [4]. On the sagittal map: Keratometric readings $>48$ Ds. SRAX $>22^{\circ}$, Superior-inferior difference $(\mathrm{S}-\mathrm{I})$ on the $5 \mathrm{~mm}$ circle $>2.5 \mathrm{Ds}$, Inferior-Superior difference (I-S) $>1.5$ Ds, Corneal astig-matism on either surface should not be higher than $6 \mathrm{D}$; otherwise, it is a risk factor, Against the rule, astigmatism is considered suspicious [5]. On the thickness map, Cone-like shape. Superior-inferior at $5 \mathrm{~mm}$ circle $>30 \mu \mathrm{m}$, Thinnest location <470 $\mu \mathrm{m}$, Thickness at pachy apex-thickness at thinnest location $>10 \mu \mathrm{m}, \mathrm{Y}$ coordinate value of the thinnest location $>-500 \mu \mathrm{m}$, Difference in thickness between both eyes at thinnest locations $>30 \mu \mathrm{m}$. On the elevation maps, Isolated island or tongue-like extension (BFS mode) on either surface, Values $>12 \mu \mathrm{m}$ within the central $5 \mathrm{~mm}$ on the anterior elevation map (BFTE mode), Values $>15 \mu \mathrm{m}$ within the central $5 \mathrm{~mm}$ on the posterior elevation map (BFTE mode) [6].

thickness $468 \mu \mathrm{m}$. Maximum corneal thickness $502 \mu \mathrm{m}$, Keratometic reading between 43.58 and 47.08 Ds. In keratoconus suspect there were 12 cases bilateral and 10 cases unilateral while in kcther were 6 cases bilateral and 4 cases unilateral. In keratoconus suspect there were 12 cases females and 10 cases males while in keratoconus there were 10 cases were females. All patients finished one and half year follow up. Patients fulfill the following criteria minimum corneal thickness 450 $\mu \mathrm{m}$, the ablation depth not more than 50 $\mu \mathrm{m}$, Keratometic reading not more than 50 Ds, spherical equivalent can be corrected by ablation of $50 \mu \mathrm{m}$, treatment is aimed at correcting mainly the cylindrical part, 
improvement of vision $\geq 2$ lines decimal visual acuity scale and Informed consent of all patients. We excluded patients with prior corneal surgery, evidence of other corneal disease, Presence of signi-ficant central corneal opacity or scarring, women who are pregnant or nursing at the time of the initial treatment, patients with Systemic collagen vascular disease, dry eyes and ocular surface disease. Preoper- rative and follow up examinations included UCVA, BSCVA, manifest refra-ction, slit lamp biomicroscopy, dilated fundus examination, corneal topography (CSO device). Before the surgery, patients were given detailed explanations of the possible implications and the surgical technique. All patients signed a written consent from in accordance with the Helsinki declaration. The ethical committee of Al-azhar University approved this study protocol.

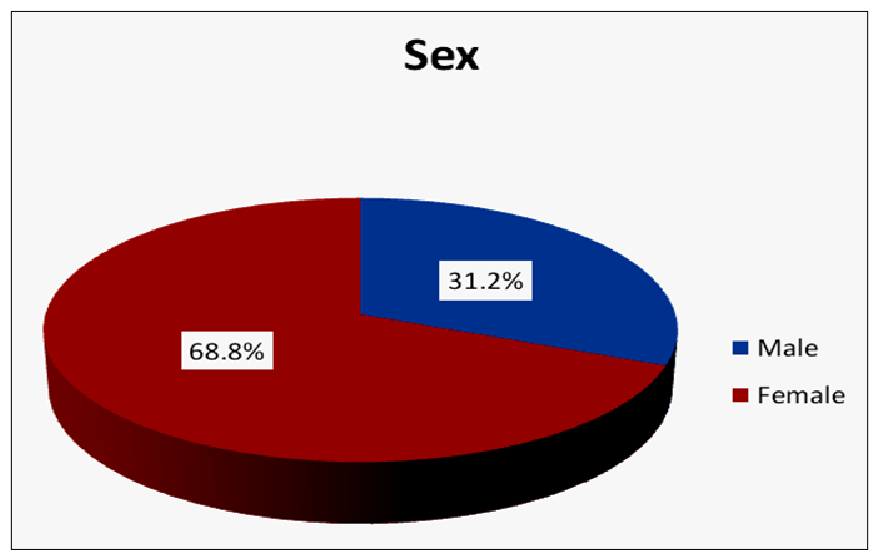

Figure (1) Distribution of patient's gender

\subsection{Surgical technique}

All patients had uneventful PRK with mitomycin $\mathrm{C}$ (MMC) followed by CXL. Thirty minutes before surgery, and Proparacaine $2 \%$ eye drops were used to anesthetize the ocular surface. The patients face was prepped with povidone-iodine $10 \%$. The eyelashes were isolated using sterile drape. Corneal epithelium was removed by PTK. Stromal ablation was done using STAR S4 IR excimer laser system (Abbott, Germany) with $6 \mathrm{~mm}$ optical zone, 1.25 $\mathrm{mm}$ transition zone and $8.5 \mathrm{~mm}$ ablation zone. MMC $0.02 \%$ was applied for 20 seconds using mirocel sponge then vigorous irrigation with BSS was done then the patient was treated with riboflavin. We used ultrasound pachymetry to locate the central and thinnest

\section{Results}

This study included fifty eyes of 32 patient $(31.2 \%)$ were male and $(68.8 \%)$ were female. the mean age of study patients was $31.2 \pm 4.2$ years old operated on between November 2014 to point. For CXL, $0.1 \%$ riboflavin with hydroxypropyl methylcellulose (Vibex Rapid; Avedrolnc, Waltham, MA, USA) applied to the corneal surface and spread with an irrigating cannula for 1.5 minutes. After the soaking, the corneal surface was washed thoroughly with balanced salt solution (BSS). A UVA beam (wave length, $365 \mathrm{~nm}$ ) $9.0 \mathrm{~mm}$ in diameter was applied to the cornea in continuous fashion in a uniform circular pattern by the CXL device (Avedrolnc). The UVA exposure was performed for 90 seconds at power of $30 \mathrm{~mW} / \mathrm{cm} 2$ (total dose, 2.7 $\mathrm{J} / \mathrm{cm} 2$ ). Patients were reviewed on the first and seventh postoperative days, and subsequently at 1 month, 3 months 6 months and one year and one and half year.

November 2016 that had simultaneous PRK and CXL in cases of suspicious keratoconus $22 \mathrm{pt}(62.5 \%)$ and early keratoconous10 pt (37.5\%). All patients finished 18 months follow up. 


\subsection{Uncorrected visual acuity (UCVA)}

The mean UCVA improved from $0.13 \pm 0.12$ preoperatively to $0.42 \pm 0.13$ at 3 months. UCVA continued to improve progressively until. As regards UCVA at last follow up we found that about 40 eye, 25 eye matched the preoperative BCVA and 15 eye gained one line while

10 eyes only lost one line little than the preoperative BCVA on decimals at the one and half year. The end of the 12 month $0.67 \pm 0.15(\mathrm{P}=0.055)$. A slight decrease at one and half year $0.6 \pm 0.14$ $(\mathrm{P}<0.001)$. The improvement was statistically significant, tab (1), fig (2).

Table (1) Comparison between preoperative and postoperative follows up as regard UCVA

\begin{tabular}{|l|c|c|c|c|c|c|c|c|c|}
\hline \multicolumn{1}{|c|}{ UCVA } & Min. & \multicolumn{1}{c}{ Max. } & \multicolumn{1}{c}{ Mean \pm SD } & P1 & P2 & \multicolumn{2}{c|}{ P3 } & P4 & \multicolumn{1}{c|}{ P5 } \\
\hline Preoperative & 0.05 & 0.50 & $0.12 \pm 0.12$ & & & & & \\
\hline After 1 Month & 0.10 & 0.50 & $0.29 \pm 0.13$ & $<0.001^{* *}$ & & & & \\
\hline After 3 months & 0.30 & 0.70 & $0.42 \pm 0.13$ & $<0.001^{* *}$ & $<0.001^{* *}$ & & & \\
\hline After 6 months & 0.40 & 0.80 & $0.67 \pm 0.15$ & $<0.001^{* *}$ & $<0.001^{* *}$ & $<0.001^{* *}$ & & \\
\hline After 1 year & 0.40 & 0.80 & $0.67 \pm 0.14$ & $<0.001^{* *}$ & $<0.001^{* *}$ & $<0.001^{* *}$ & $<0.001^{* *}$ & \\
\hline After 1.5 year & 0.30 & 0.70 & $0.6 \pm 0.14$ & $<0.001^{* *}$ & $<0.001^{* *}$ & $<0.001^{* *}$ & $<0.001^{* *}$ & $<0.001^{* *}$ \\
\hline
\end{tabular}

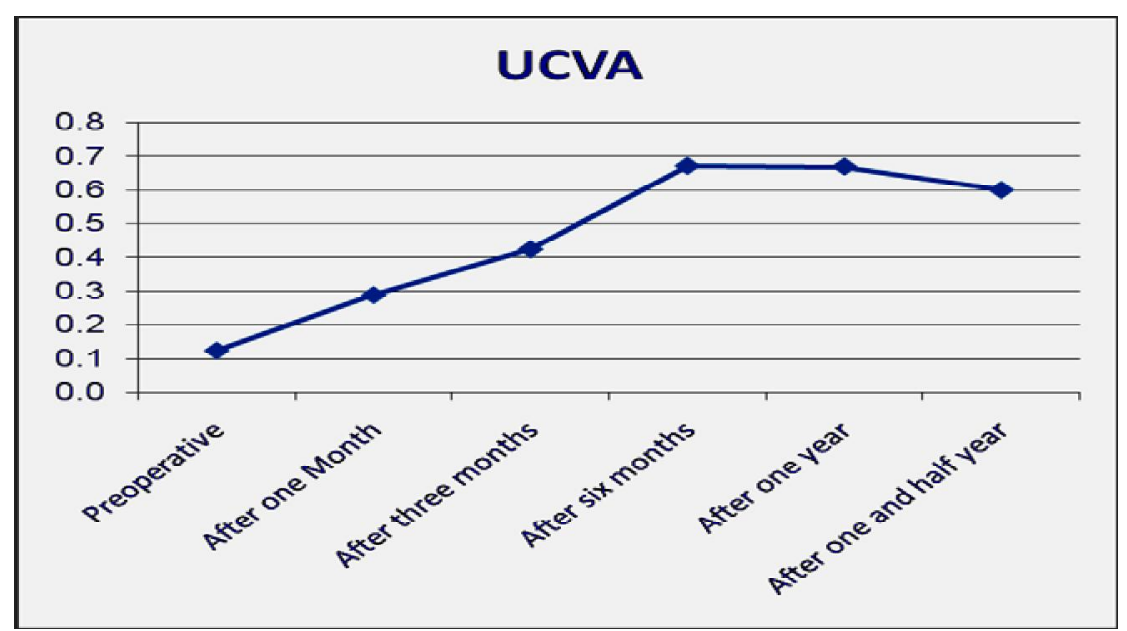

Figure (2) Comparison between preoperative and follow up as regards UCVA.

\subsection{Best corrected visual acuity (BCVA)}

The preoperative mean BCVA 0.65 \pm 0.17 preoperatively showed an significant change (P 0.001) at 3 month postoperatively $(0.58 \pm 0.17)(\mathrm{P}<0.001)$ and continued with improvement at 6 month $0.75 \pm 0.17$ and stabled over the followup period, reaching $0.75 \pm 0.16$ at 12 months $(\mathrm{P}=0.001)$ and $0.75 \pm 0.16$ at one and half year $(\mathrm{P}=0.001)$ respectively. Ten eyes $(20 \%)$ showed no improvement in BCVA in lines, thirty eyes $(60 \%)$ gained one line, $10 \%$ gained two lines, $10 \%$ gained 3 lines, BCVA. No eyes lost any lines in BCVA at the one and half year follow-up, tab (2), fig (3).

Table (2) Comparison between preoperative and postoperative follows up as regard BCVA

\begin{tabular}{|l|c|c|c|c|c|c|c|c|}
\hline \multicolumn{1}{|c|}{ BCVA } & Min. & Max. & Mean \pm SD & P1 & P2 & P3 & P4 & P5 \\
\hline Preoperative & 0.40 & 0.90 & $0.65 \pm 0.17$ & & & & & \\
\hline After 1 Month & 0.30 & 0.80 & $0.47 \pm 0.16$ & $<0.001^{* *}$ & & & & \\
\hline After 3 months & 0.40 & 0.80 & $0.58 \pm 0.17$ & $<0.001^{* *}$ & $<0.001^{* *}$ & & & \\
\hline After 6 months & 0.40 & 0.90 & $0.75 \pm 0.17$ & $<0.001^{* *}$ & $<0.001^{* *}$ & $0.024^{*}$ & & \\
\hline After 1 year & 0.40 & 0.90 & $0.75 \pm 0.16$ & $<0.001^{* *}$ & $<0.001^{* *}$ & $0.024^{*}$ & 1.000 & \\
\hline After 1.5 year & 0.40 & 0.90 & $0.75 \pm 0.16$ & $<0.001^{* *}$ & $<0.001^{* *}$ & $0.024^{*}$ & 1.000 & 1.000 \\
\hline
\end{tabular}




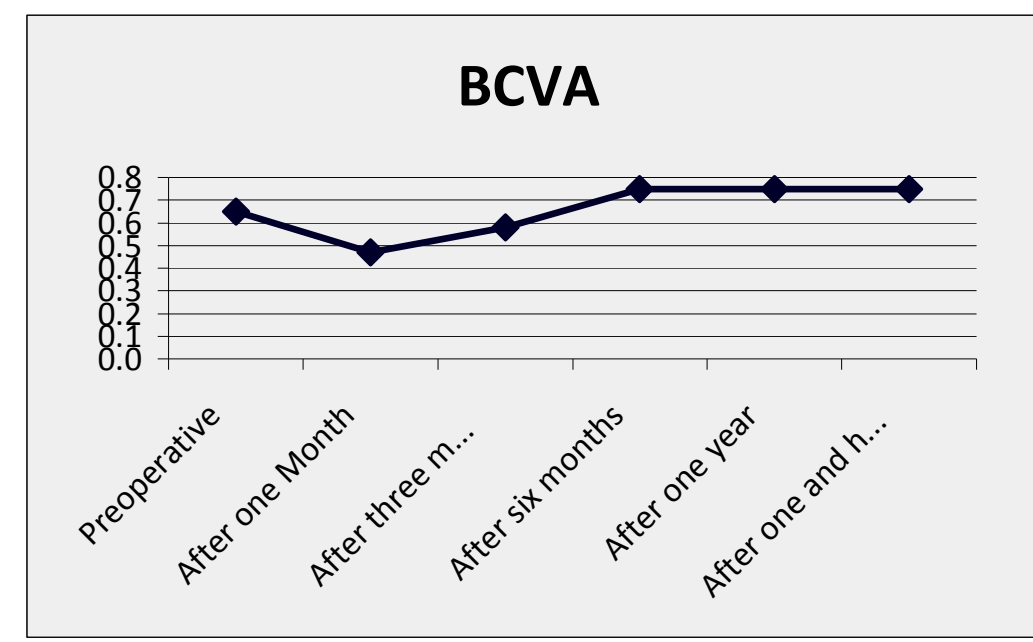

Figure (3) Comparison between preoperative and follow up as regards BCVA

\subsection{Spherical equivalent (SE)}

The mean spherical equivalent (SE) refraction decreased significantly from $3.26 \pm 2.78 \mathrm{D}$ preoperatively to $-0.92 \pm$ $0.46 \mathrm{D},-1.08 \pm 0.4 \mathrm{D}$ and $-1.47 \pm 0.38$ at 3 months $(\mathrm{P}<0.001), 12$ months and one and half year $(\mathrm{P}<0.001)$ respectively. The changes in spherical equivalent (SE) were statistically significant at 3 months SE improved (decreasing) with no significant changes but at 1 year slight increasing and at one year and half more increaseing in SE occurred, tab (3), fig (4).

Table (3) Comparison between preoperative and postoperative follows up as regards SE

\begin{tabular}{|l|c|c|c|c|c|c|c|c|}
\hline \multicolumn{1}{|c|}{ SE } & Min. & Max. & Mean \pm SD & P1 & P2 & P3 & P4 & P5 \\
\hline Preoperative & -2.50 & -6.50 & $-3.26 \pm 2.78$ & & & & & \\
\hline After 1 Month & -0.50 & -1.75 & $-0.99 \pm 0.39$ & $<0.001^{* *}$ & & & & \\
\hline After 3 months & -0.25 & -1.75 & $-0.92 \pm 0.46$ & $<0.001^{* *}$ & $<0.001^{* *}$ & & & \\
\hline After 6 months & -0.25 & -1.75 & $-0.94 \pm 0.45$ & $<0.001^{* *}$ & $0.002^{* *}$ & $0.024^{*}$ & & \\
\hline After 1 year & -0.25 & -1.75 & $-1.08 \pm 0.4$ & $<0.001^{* *}$ & $0.013^{*}$ & $<0.001^{* *}$ & $<0.001^{* *}$ & \\
\hline After 1.5 year & -0.50 & -2.00 & $-1.47 \pm 0.38$ & $<0.001^{* *}$ & $<0.001^{* *}$ & $<0.001^{* *}$ & $<0.001^{* *}$ & $<0.001^{* *}$ \\
\hline
\end{tabular}

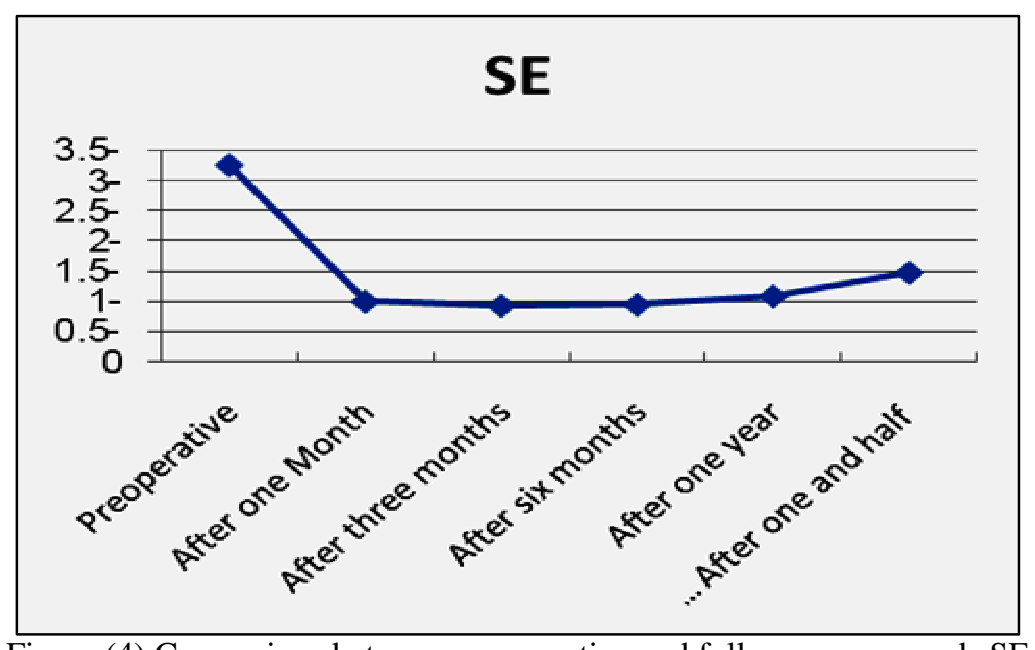

Figure (4) Comparison between preoperative and follow up as regards SE

\subsection{Refraction of cylinder}

The mean manifest refraction cylinder decreased from $-2 \pm 0.86 \mathrm{D}$ preoperatively to $-0.61 \pm 0.36,-0.82 \pm 0.27 \mathrm{D}$ and $-1.14 \pm 0.23$ at 6 months $(\mathrm{P}<0.001)$, 12 months $(\mathrm{P}=0.012)$ and one and half year $(\mathrm{P}<0.001)$ respectively, tab $(4)$, fig 
(5) The changes (decreasing) in manifest refraction of cylinder were significant at one months, 6 months while increased slightly at 12 months and more signific- antly at one and half year respectively. Slight regression about $54 \%$ of the eyes had Astigmatism by $\mathrm{K}$ Reading within 1.0D. None of the eyes had $>2.0 \mathrm{D}$.

Table (4) Comparison between preoperative and postoperative follow up as regards cylinder :

\begin{tabular}{|c|c|c|c|c|c|c|c|c|}
\hline Cylinder & Min. & Max. & Mean \pm S & P1 & P2 & P3 & P4 & P5 \\
\hline Preoperative & -1.00 & -3.50 & $-2 \pm 0.86$ & & & & & \\
\hline After 1 Month & 0.00 & -1.50 & $-0.61 \pm 0.36$ & $<0.001^{* *}$ & & & & \\
\hline After 3 months & 0.00 & -1.50 & $-0.61 \pm 0.36$ & $<0.001 * *$ & 1.000 & & & \\
\hline After 6 months & -0.50 & -1.50 & $-0.75 \pm 0.32$ & $<0.001 * *$ & $<0.001 * *$ & $<0.001 * *$ & & \\
\hline After 1 year & -0.50 & -1.25 & $-0.82 \pm 0.27$ & $<0.001 * *$ & $<0.001 * *$ & $<0.001 * *$ & $0.012 *$ & \\
\hline After 1.5 year & -0.75 & -1.50 & $-1.14 \pm 0.23$ & $<0.001 * *$ & $<0.001 * *$ & $<0.001 * *$ & $<0.001 * *$ & $<0.001 * *$ \\
\hline
\end{tabular}

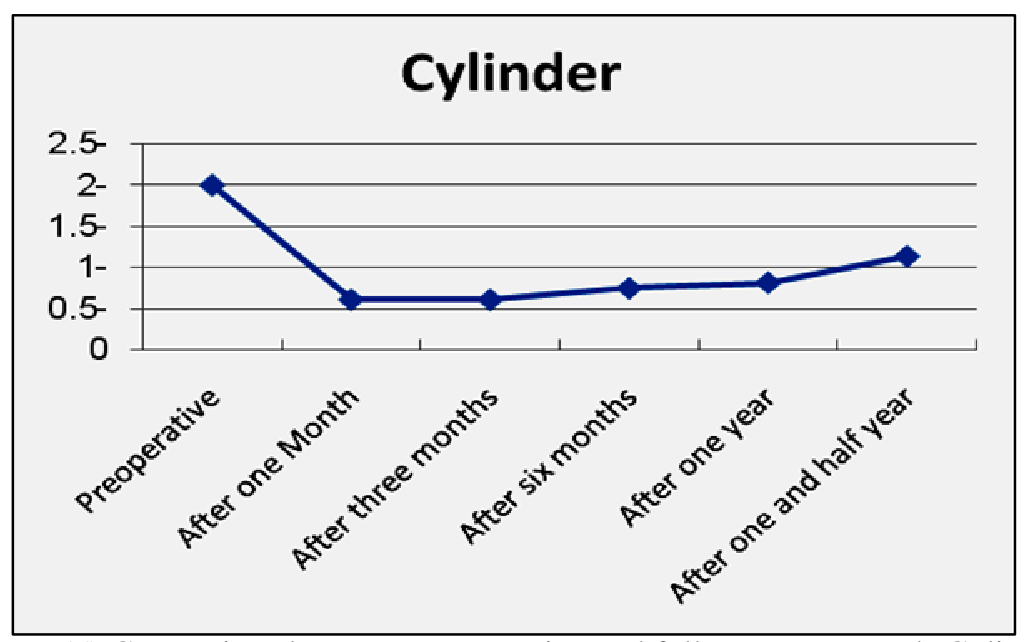

Figure (5) Comparison between preoperative and follow up as regards Cylinder

\subsection{Keratometry}

The mean average K-value (mean K) decreased from $44.47 \pm 1.16$ to 42.16 $\pm 2.36,42.34 \pm 2.23$ and $42.37 \pm 2.22 \mathrm{D}$ at 3,12 and 1.5 years post operatively, a statistically significant reduction $(\mathrm{P}<0.01)$, fig (6). The mean steepest keratometry ( $\mathrm{K} \max$ ) were $45.49 \pm 1.25 \mathrm{vs} 43.23 \pm 2.33$, $43.28 \pm 2.34$ and $43.32 \pm 2.33 \mathrm{D}$ at 3.12 and

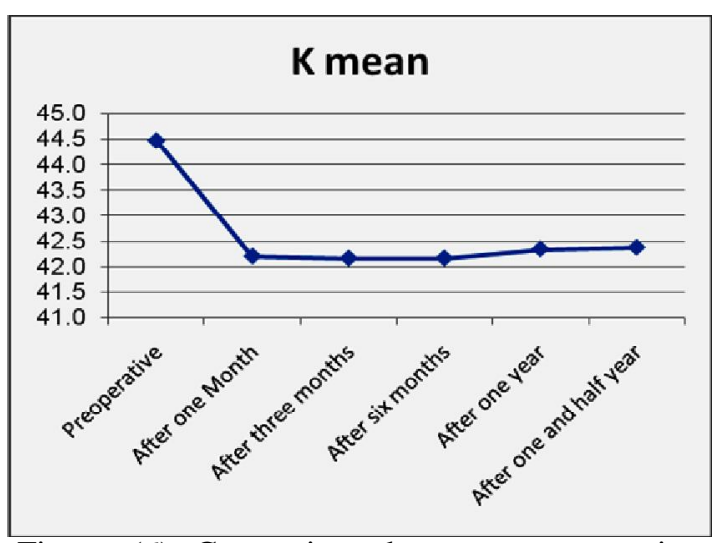

Figure (6) Comparison between preoperative and follow up as regards mean $\mathrm{K}$
1.5 years post operatively ( $\mathrm{P}<0.001)$. Keratometric values appeared stable postoperatively, with no evidence of progression in $\mathrm{K}$ max over the follow up period. Postoperatively, a statistically significant reduction $(\mathrm{P}<0.01)$, fig $_{\mathrm{s}}(7$ \& 8).

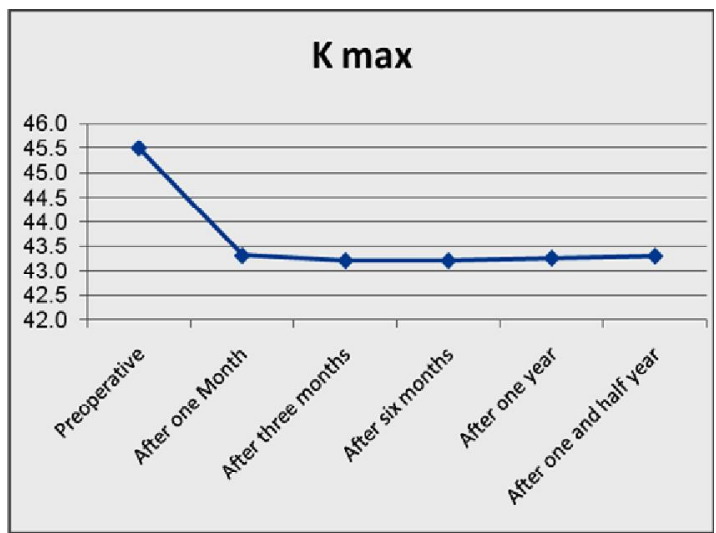

Figure (7) Comparison between preoperative and follow up as regards $\mathrm{K}$ max 


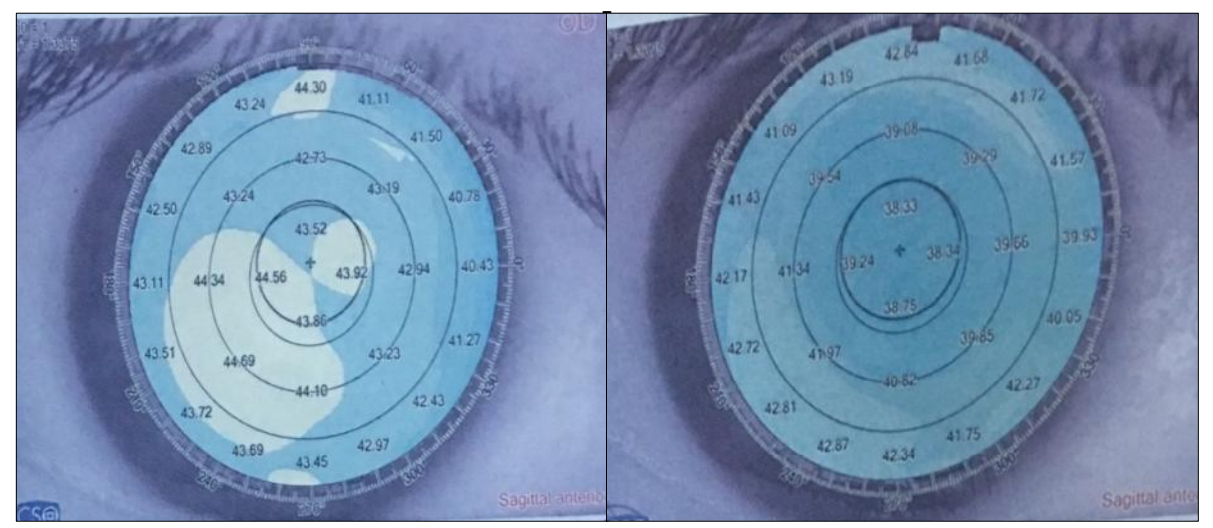

Figure (8) Right Pentacam image shows corneal inferior steepening before PRK extra and Left Pentacam image shows flattening of the steep cornea after ablation within safety till last follow up period.

The early complications including post-operative pain, no significant pain on postoperative day 1 was reported in $45 \%$ of the cases, while there were moderate pain in $25 \%$, and severe pain in $30 \%$ of patients. This is most likely due to removal of epithelium. The patients were improved gradually by week after talking systemic nonsteroidal anti-inflammatory drugs (diclofenac sodium $50 \mathrm{mg}$ twice daily). Delayed epithelial healing was reported if the epithelium did not heal by day 5 post procedure, epithelial healing time occurred in $75 \%$ of cases by day $4,15 \%$ by day 5 , and $10 \%$ delayed epithelial healing by day 10 . The patients were improved completely after they treated with lubrication (carboxymehyl cellulose $1 \%$, sodium hyaluronate, polyvidone 50 $\mathrm{mg}) 6$ times daily and complete healing after one week later, fig. (9). Late complications including stromal haze, Transient stromal haze was noted in about $10 \%$ of the cases ( 5 eyes, with two eyes grade 1 , two eyes grade 2 and one eye grade 3 ).
Only the eye with grade 3 haze was felt to have any possible impact on vision, although this eye still had a vision of 0.15 decimal compared to 0.3 preoperatively, and was continuing to regress and visual improvement till it completely relived by 6 month after surgery to reach 0.2 till last follow up while eyes with grade one resolved by 1 month and those with grade 2 improved by month 4 after procedures, fig. (10). Subepithelial corneal scar was noted in about $4 \%$ ( 2 eyes) with persistent scar beyond six months that didn't respond to the various interventions weren't felt to have any possible impact on vision as the scar were paracentral in both, although one eye still had a vision of 0.4 decimal compared to 0.3 preoperatively with improvement one line but the other one still had a vision of 0.5 decimal compared to 0.5 preoperatively, fig. (11). However none of these cases necessitated PKP. No ectatic progression no corneal melting, no infectious keratitis.

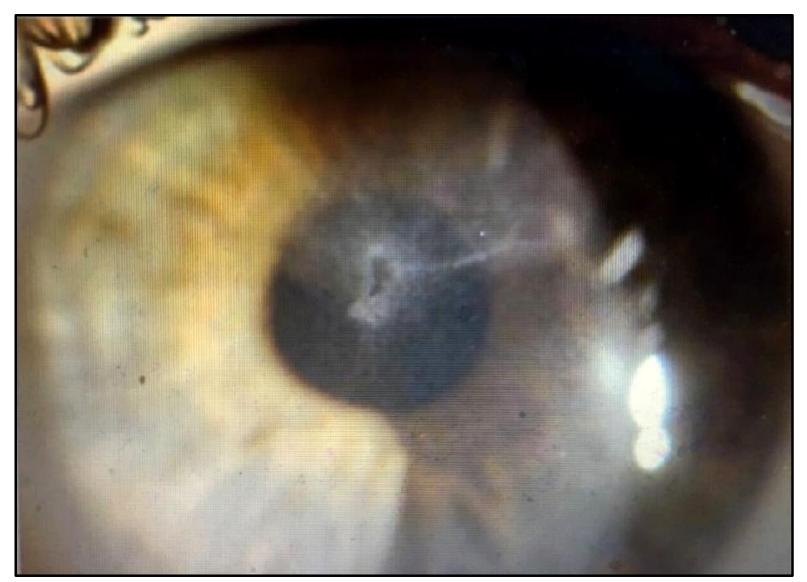

Figure (9) Delayed epithelial healing, stromal haze 


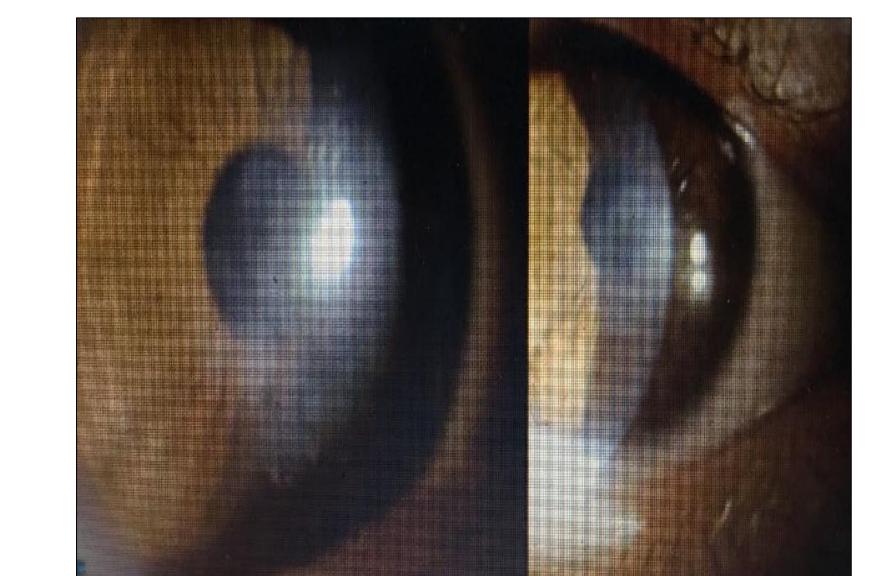

Figure (10) PRK-like haze, 1 year after improved with steroids

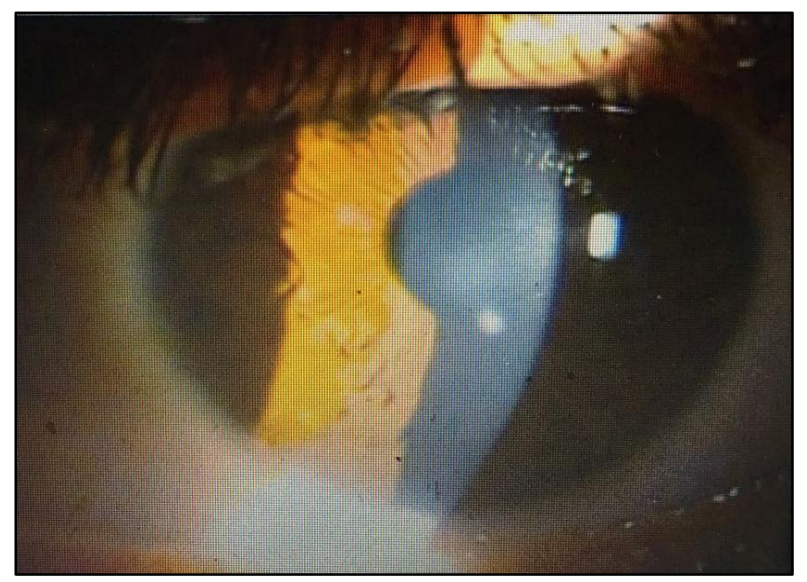

Figure (11) Faint scar and white spot

\section{Discussion}

The goal of simultaneous custom PRK and CXL is to offer keratoconus patients corneal stability as well as functional vision. Reaching functional vision involves improving UCVA, BCVA, and corneal irregularity so that patients are less dependent on contact lenses to achieve better quality of vision. Custom PRK is a predictable and effective technique to achieve remodeling of the corneal surface and rehabilitation of refractive impairment. CXL is capable of stabilizing the corrected cornea and inhibiting keratoconic progression. In this study we utilized non-topographic aspheric profiles derived from refraction and topography, combined with simultaneous CXL. In contrast, current treatment strategies use topographically derived treatment profiles with the intention of correcting aberrations. The use of topographic profiles however, assume that the shape change in the ecstatic cornea predictably responds to ablation shape. However, there are several potential causes of unpredictable response to topographic planning. Firstly, the use of topographic profiles ignores the biomechanical effects of such ablations in compromised cornea [7]. Secondly, the actual tissue ablation rate may differ in keratoconic tissue, with unexpected topographic effects. Thirdly, the measured and ablated surfaces differ unless a trans epithelial therapy is used, since the epithelial profile in keratoconus is irregular [8]. Finally and most significantly, cross linking itself has progressive effects on keratoconic topography, which are not accounted for $[9,10]$. Our rationale for non-topographic aspheric astigmatic profiles was minimize stromal ablation, while preserving beneficial effects of combining PRK and CXL. A transepithelial treatment was chosen to obtain a corrective effect based on the differential epithelial profile around 
the cone, which has been shown to have a 'doughnut' distribution, thus the masking effect of the epithelium would tend to regularize the cone [11]. Notably, combined excimer laser ablation and CXL may also have a beneficial effect on keratoconus independent of topographic or refractive correction. This has been demonstrated in a recent series by Kymionis et al., where PTK epithelial removal for CXL resulted in superior visual outcomes compared to mechanical removal [12]. Although MMC in keratoconic PRK has been used, we felt that CXL induced keratocytes depopulation offsets the need for chemotherapy, particularly as the transepithelial treatment causes less haze [13]. Although we had initial concerns regarding potential haze, in this study PRK treatment was planned based on the patient's corneal thickness. Limited treatments of up to $50 \mu \mathrm{m}$ were performed; the ablation depth was mapped out by modifying the target correction. CXL treatment was performed immediately following the ablation we demonstrated a rapid and significant improvement. As regards UCVA at last follow up we found that about 40 eye improved, 25 eye matched the preoperative BCVA and 15 eye gained one line while 10 eyes only lost one line little than the preoperative BCVA on decimals at the one and half year in comparison to preoperative BCVA. The efficacy index (postoperative mean decimal UDVA divided by preoperative mean decimal BCDVA) was 0.65 at three months after surgery, improved to 1.03 at 6 months, and continued without significant change at 12 months, but reaching 0.92 with slight decrease At one and half year matched. The standard deviation of UDVA was $0.12 \pm 0.12$ preoperatively and $0.67 \pm 0.14$ postoperatively with progression and stability over twelve months follow-up period. At one and half year slight regression occurred reaching $0.6 \pm 0.14$.As regards BCVA at last follow up we found that about Ten eyes (20\%) showed no improvement in BCVA in lines, 25 eyes $(60 \%)$ gained one line, $10 \%$ gained two lines, $10 \%$ gained 3 lines, BCVA. no eyes lost one line in
BCVA at the one and half year follow-up in comparison to preoperative BCVA due to $3^{\text {rd }}$ degree haze and sub epithelial scar affecting cornea after procedure. The safety index (postoperative mean decimal BCVA divided by preoperative mean decimal BCDVA) showed a progressive improvement from 0.89 at one month, reaching 1.15 at 6 months and settling at 1.25 at 12 months and remained stable at one and half year. Likewise, the standard deviation of CDVA was $0.65 \pm 0.17$ preoperatively and $0.75 \pm 0.16$ postoperatively with progression and stability over the follow-up period ( $\mathrm{P}=0.001)$. As regard astigmatism about $54 \%$ of the eyes had Astigmatism by K-Reading within 1.0 $\mathrm{D}$ and $63 \%$ of the eyes had Astigmatism by Refraction within $1.0 \mathrm{D}$. No eyes had > $2.0 \mathrm{D}$. A reduction in topographic astigmatism was observed after 18 months follow-up, with no significant improvement in visual outcomes. Corresponding to the positive change of UCVA and BCVA, we found a reduction of myopia. Probably due to regularization of the cornea, subjectively accepted cylinder was higher after CXL+ PRK in many cases. The underlying reason for the positive changes in visual acuity and refraction is the observed corneal flatting, as shown by the reduction of maximum and minimum keratometry values. Complications were found in five eyes of 50 eyes. Only one eye lost one line of BCVA due to prolonged central Descemet's folds and stromal edema up to 6 months, while other eyes with grade one stromal haze resolved by 1 month and those with grade 2 improved at 4 months after procedures. The major limitation of the study was the absence of a control group. We did not feel that a retrospective control group of CXL alone would be superior to comparison of existing large CXL series. A future study comparing topographic to nontopographic treatment is planned. Notwithstanding these issues, we feel that this technique shows promise as a preliminary intervention in suspect and early keratoconic patients. In addition, the follow-up period in our study was 18 months and due to the reports of progressive excessive corneal flattening $[14,15]$ which may lead 
to hyperopic shift up to several years after CXL [16] further studies with longer follow-up periods are needed to further evaluate the long-term outcomes of this technique.

\section{Conclusion}

The Combined non-topography-guided PRK and CXL is an effective procedures for correcting mild refractive error and improving visual acuity and safety option for arresting the ectatic progression, with improvement of corneal keratometric values in patients with suspicious and early keratoconus provided that we adhere to the selection criteria of that we recommend. However, future studies with larger cohorts of patients and longer follow-up periods are needed to determine the safety, efficacy, and stability of such procedure.

\section{References}

1. Romero-Jiménez, M., SantodomingoRubido, J., Wolffsohn, J., Keratoconus W., A review. Cont Lens Anterior Eye 2010; 33 (4): 157-166.

2. Maeda, N., Klyce, S., Smolek, M., Thompson, H. Automated keratoconus screening with corneal topography analysis. Investigative Ophthalmology \& Visual Science 1994; 35: 2749-2757.

3. Matalia, H., Swarup, R., Imaging modalities in keratoconus. Indian $\boldsymbol{J}$ Ophthalmol. 2013; 61 (8): 394-400.

4. Sinjab, M. Quick guide to the management of keratoconus: A systematic stepby-step approach, Springer, Germany, 2012, p. 26

5. Chan, C., Sharma, M., Boxer Wachler, B. Effect of inferior-segment intacs with and without C3-R on keratoconus. $\boldsymbol{J}$ Cataract Refract Surg. 2007; 33: 75-80.

6. Rathi, V., Mandathara, P., Dumpati, S. Contact lens in keratoconus. Indian J Ophthalmol. 2013; 61: 410-415.

7. Dupps, W., Wilson, S. Biomechanics and wound healing in the cornea. $\operatorname{Exp}$ Eye Res. 2006; 83: 709-720.

8. Stojanovic, A., Zhang, J., Chen, X., Nitter,T.,Chen,S.,Wang,Q.Topographyguided transepithelial surface ablation followed by corneal collagen crosslinking performed in a single combined procedure for the treatment of keratoconus and pellucid marginal degeneration. J Refract Surg. 2010; 26: 145152.

9. Raiskup-Wolf, F., Hoyer, A., Spoerl, E., Pillunat, L. Collagen cross-linking with riboflavin and ultraviolet-A light in keratoconus: Long-term results. J Cataract Refract Surg. 2008; 34: 796-801

10. Tu, K., Aslanides, I. Orbscan II anterior elevation changes following corneal collagen cross-linking treatment for keratoconus. J Refract Surg. 2009; 25: 715-722.

11. Reinstein, D., Gobbe, M., Archer, T., Silverman, R., Coleman, D. Epithelial, stromal, and total corneal thickness in keratoconus: Three-dimensional display with artemis very-high frequency digital ultrasound. J Refract Surg. 2010; 26: 259-271.

12. Kymionis, G., Grentzelos, M., Kounis, G., Diakonis, V., Limnopoulou, A., Panagopoulou, S. Combined transepithelial phototherapeutic keratectomy and corneal collagen cross-linking for progressive keratoconus. Ophthalmology. 2012; 119(9): 1777-1784

13. Aslanides, I., Padroni, S., ArbaMosquera, S., Ioannides, A., Mukherjee, A. Comparison of single-step reverse transepithelial all-surface laser ablation (ASLA) to alcohol-assisted photorefractive keratectomy. ClinOphthalmol. 2012; 6: 973-980.

14. Santhiago, M., Giacomin, N., Medeiros, D., Smadja, C., Bechara, S. Intense early flattening after corneal collagen cross-linking. J of Refractive Surgery 2015; 31 (6): 419-422.

15. Greenstein, S., Fry, K., Hersh, P. Effect of topographic cone location on outcomes of corneal collagen cross- 
linking for keratoconus and corneal ectasia. J of Refractive Surgery 2012; 28 (6): 397-405.

16. Kymionis, G., Tsoulnaras, K., Liakopoulos, D., Paraskevopoulos, T.,
Kouroupaki, A., Tsilimbaris, M. Excessive corneal flattening and thinning after corneal cross-linking: Singlecase report with 5-year follow-up. Cornea 2015; 34 (6): 704-706. 